\title{
Analysis and Research on Learning Behavior Based on LMS
}

\author{
Shangyu $\mathrm{Wu}^{1}$, Junming $\mathrm{Ye}^{2, *}$, Zhifeng Wang ${ }^{3, *}$, Daxiong Luo ${ }^{4}$ and Rong Zhao ${ }^{5}$ \\ ${ }^{1,2,4}$ School of Computer, Central China Normal University, Wuhan 430079, China \\ ${ }^{3,5}$ School of Educational Information Technology, Central China Normal University, Wuhan 430079, China \\ *Corresponding author: jmye@ mail.ccnu.edu.cn, zfwang@mail.ccnu.edu.cn
}

\begin{abstract}
With the rapid development of Internet technology, big data has become more and more frequently mentioned. How to use the latest technology to dig valuable information from big data has become a hot topic. In this paper, online learning analysis platform based on education big data integrated the acquisition and analysis of students' data. It allowed teacher to teach online, students and students to learn online. At the meantime, it would gather students' data automatically and filter out effective data based on some requirements. Finally, it would analyze data using learning analysis technology and give teacher a feedback of the analysis results. This system would assist teachers more targeted teaching activities.
\end{abstract}

Keywords-education big data; online learning; learning analysis

\section{INTRODUCTION}

This paper mainly introduces how to build an online learning platform which integrates learning, management and monitoring, and presents detailed analysis results combined with statistics, machine learning and other knowledge. When doing system development from the perspective of objectoriented, it needs to consider three models: function model, object model, dynamic model, the learning analysis system using methods of object-oriented software development, and use the UML notation system modeling, in order to facilitate a better understanding of complex learning analysis system.

The functional model uses the use case diagram in UML to describe the functionality of the system from the perspective of the users [1]. To build function model first needs to identify the actors. Actors are an entity that not belongs to system, and during the execution, the actors input to the system and trigger the execution of the system [2]. It is important to note that actors are usually not fixed and can be the role of persons or things when interacting with the system. Actors can play multiple roles simultaneously with different interactions with the system. According to the functional structure of the learning analysis system, the use case diagram of this system can be divided into four layers: system layer, course layer, section layer and knowledge point layer [3-5].

On the system layer, the administrators have the function such as curriculum management, student management, teacher management, curriculum management functions including course to add or delete, the distribution of the classroom, the student management functions including students to add or delete, and so on, teachers management functions including teachers to add or delete, and other functions [6]; Students can select course, see the list, and select course function allows students to choose a course of a teacher, see the course list functionality allows students to see the selected course; Teachers can see the course list. Teachers are assigned by the administrators directly, so teachers don't need to choose courses, check the course list functionality allows teachers to see what they need to teach.

On the course layer, students have functions of check the section list, check the homework, submit homework, and so on [7-9]. Check the section list functionality allows students to see the sections teachers set, check the homework allows students to see sections of homework, including information such as when to start or stop, submit homework allows students to submit a job function within a given time to teacher in the form of attachment; Teachers have check section list, check the homework, add operation, delete operation, corrects students' papers, add sections, delete sections, update the weight, and other functions, check section list functionality allows teachers to check the sections they add, update the weights allows teachers to update the weight of each section, simplifying the final accounting and statistics.

On the section layer, students can check the list of knowledge points, check the personal grade and so on [10-13]. Check the list of knowledge points list functionality allows students to view the knowledge point set up by teachers, check the personal grade functionality allows students to view the grades of classroom lecture and homework; Teachers can check the list of knowledge points, add knowledge point and remove knowledge point, update the weights, scoring, check the summary report and other functions, check the list of knowledge points functionality allows teachers to check the knowledge point they add, update the weights functionality allows teachers to update the weight of class lecture, questionanswer process in class, attendance, homework, experiment, review or preview learning point, scoring functionality allows teachers to score points of the course students selected, check the summary report functionality allows teachers to see the section summary report, including the score statistics and other information.

On the knowledge point layer, students can fill the questionnaire, BBS posts, view video, exam, and so on [14]. Fill in the questionnaire functionality allows students to fill the questionnaire set by teachers, exam functionality allows students to participate in three exams in each knowledge set by 
teachers, including the exam of video, PPT exam, the exam; Teachers can do BBS posts, BBS return card, view video, add video, delete video, set up the exam, add questions, delete questions, statistics data, and so on. Setting exam functionality allows teachers to set up to three exams for each knowledge point, including the exam of video, PPT exam, the exam [15]. Add questions function allows teachers to add questions to question bank, delete questions function allows teachers to delete questions in question bank, statistics data function allows teachers to check the results of the data from students using machine learning methods, such as form, line chart, histogram, etc.

\section{LEARNING ANALYSIS SySTEM DESIGN}

\section{A. System Architecture}

The architecture design of the system is shown in FIGURE 1.

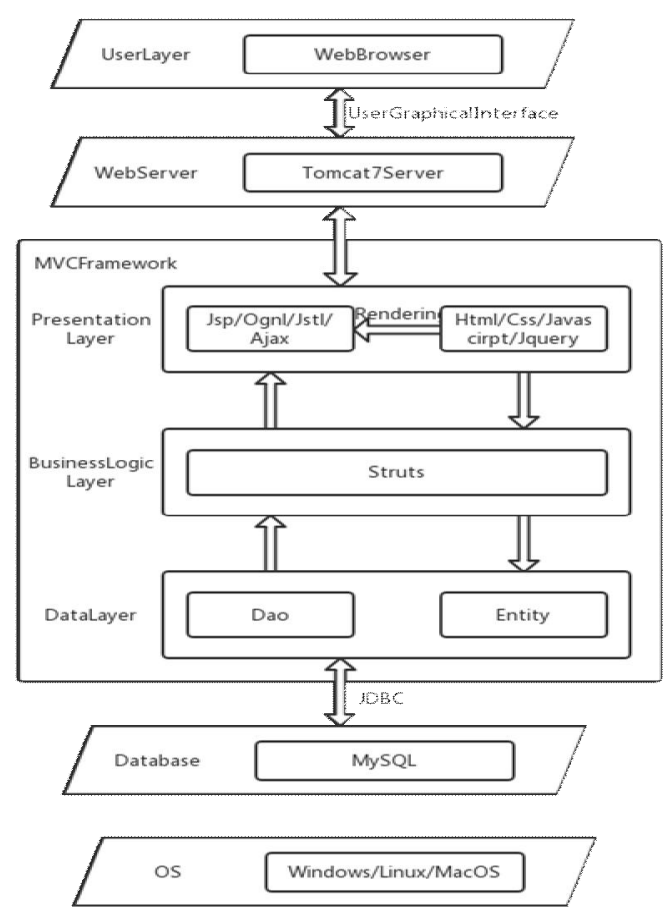

FIGURE I. SYSTEM ARCHITECTURE DIAGRAM

The overall architecture of the system is divided into user layer, presentation layer, business logic layer, data layer and data objects passing between layers. The system based on the MVC framework is divided into three layers: the presentation layer, the business logic layer and the data layer. Among them, the use of Struts is responsible for the separation of MVC, control business jump; use components to provide support for data query updates, and separate each entity Entity. Each layer is described in detail below:

- User Layer: The user layer is simply the level used to interact with the user and display information from the system to the user.
- Presentation Layer: The presentation layer mainly refers to the appearance of the page and the part of the auxiliary page interaction. The presentation layer to implement the interactive interface by tools such as Jsp/Ognl/Jstl/Ajax, is responsible for receiving a request (Request) and transmission response (Response), and the $\mathrm{Html} / \mathrm{Css} / \mathrm{Javascript/Jquery}$ was used to render the page, makes the interface more humane.

- Business Logic Layer: The business logic layer deals with the core business logic of the system. Struts is responsible for the business logic control of the system and the Http request generated by the client. The HTTP request is mapped to the Action class through the XML configuration file, and the HTTP request is handed over to the corresponding Action class. The Action class is responsible for invoking the appropriate Dao layer components to satisfy updates to the database.

- Data Layer: The data layer objects the database, forms the entity (Entity), maps the entity and the database table, and the Dao layer component handles the request operation of the upper layer to the database, and returns the processing result.

\section{B. Module Design}

According to the analysis of system module division, can be divided into user module, module assembly, module, knowledge section module components, operation module, questionnaire module components, BBS module module, video module, exam module. FIGURE 2 is the UML deployment diagram of the system.

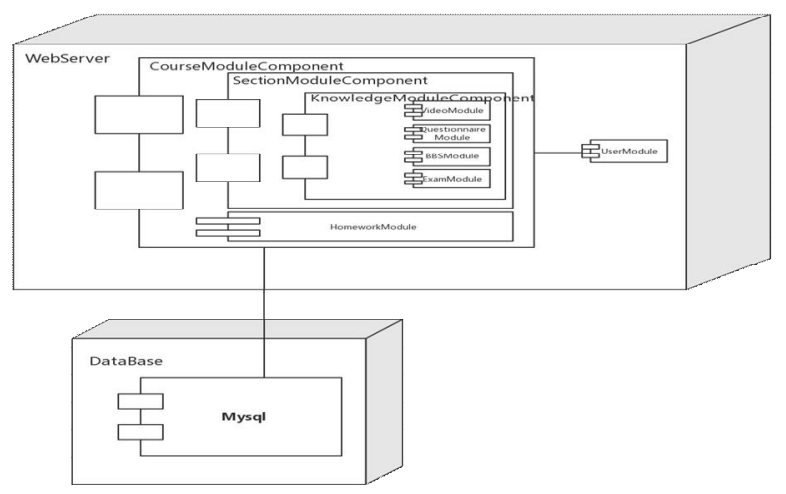

FIGURE II. SYSTEM DEPLOYMENT DIAGRAM

The following describes the design of the system in accordance with the division of module components.

- User module component design

The system has three roles, namely, students, teachers, administrators. The system takes the student and the teacher part as the main role, the administrator as the auxiliary role. Administrators can be responsible for user management, curriculum management and other functions. The students are mainly online learning, and teachers are mainly concerned with managing students.

- Module design of course module

This system curriculum module component mainly includes the curriculum choice, the curriculum content review, the 
curriculum content renewal and so on function, simultaneously also includes the operation module component.Students may choose a course based on different classes, such as selecting a teacher's class in the course operating system, or deleting the selected courses. At the same time, the existing data will be clearly deleted. Students can view their selected course content, namely, section list.Teachers are not able to choose courses compared to students, but they still have the function of looking at the contents of the course, that is, the list of sections. Teachers from different classes in the same class can personalize their course content, i.e. adding sections and deleting sections. Teachers can add weights to the added sections so that they can calculate the total score of the course.Administrators can add or delete courses, but cannot edit the course content. You can also set the number of classes for each course and assign the teacher to the assigned class.

\section{- Section module component design}

This system section module component mainly includes the section content, the view, the section content renewal and so on.Students can view sections, i.e., lists of knowledge points. Students can also view the section on personal achievements, including the classroom and classroom to answer questions, attendance, homework, preview, review, experimental evaluation.Teachers can check the contents of the section, that is, the list of knowledge points, while you can manage the contents of the section, that is, add knowledge points, delete knowledge points. Teachers can activities (classroom lectures, classroom attendance, homework, answer questions, exam, preview, review comments) add to set the weights, in order to calculate the total score of the student section. The teacher can grade the student's activity for the course.

\section{- Component design of knowledge point module}

The knowledge point module component mainly includes data statistics and so on. It includes questionnaire module, component module, exam module and video module.The function of data statistics is limited to the teachers. This function will analyze the data in the module under the knowledge point module, and display the results in the form of charts. Specific analysis and design, see fifth sections.

\section{- Job module component design}

The job module components are included in the course module components, because assignments are arranged in sections, a section can have multiple jobs, and a job belongs to only one section. Assignments include job titles, assignments, sections, assignments, start times, end times, assignments, and so on. Students can view the assignments in each section, as well as all the content of each job. Students can submit their assignments in the form of attachments, and can also add note information. The student may download the submitted attachment to the local for review, or delete the submitted job attachment and resubmit the job. Teachers can look at the assignments already laid out in each section, and also look at all of the content of each job. Teachers can add new jobs, or delete jobs that have already been assigned. Teachers can download attachments that have been assigned to work, or remove attachments to already assigned jobs. The teacher can also correct each assignment. In order to facilitate the correction, the teacher can view each student's submission, that is, download the attachment submitted by the student and view the student's memo information. Teachers can also change their grades for already corrected assignments.

\section{- Module design of questionnaire module}

The questionnaire module component is included in the knowledge point module component, and the knowledge point and the questionnaire only correspond, that is, a knowledge point contains only one questionnaire. The questionnaire includes three choice questions: class time evaluation, in class teaching evaluation, extracurricular teaching evaluation, a fill in the blank question: learning methods input. Multiple-choice questions can be chosen with less satisfaction, more satisfaction, and great satisfaction. The method of filling in the blanks needs to be filled with the solutions made by students when they are confronted with difficulties in the course of learning. Students at each point of knowledge can fill in a questionnaire, and fill out the questionnaire, you can not modify or delete. Teachers in the data statistics function, you can see each student fill in the questionnaire information, you can also see each choice of statistical information.

\section{- $\quad$ BBS module design}

The BBS module component is contained in the knowledge point module component, and each knowledge point contains a BBS belonging to the knowledge point. Students, teachers can post, reply to the post, the first speaker was identified as the landlord. Teachers in the release and reply to the post will be marked as a teacher.

\section{- Design of video module component}

The video module component is contained in the knowledge point module component, and each knowledge point contains at most one video belonging to the knowledge point. The video playback uses the video tag in Html5. Students can play video at any time, play back to any place, play video, or play video in full screen. The teacher can manage video on the basis of the video function of the students, that is, adding video and deleting video.

\section{- Component design of exam module}

The exam module component is included in the knowledge point module component, and each knowledge point contains three exams, namely, video exam, classroom exam, and knowledge point quiz. Each exam includes the beginning of the exam, the end of the exam, and the list of topics. The subjects of the exam are all from the question bank, and they are all multiple-choice questions. The problems in the problem library are added manually. Students can look at the basic information that has been created and answer the exam. Students have already answered questions, and students are not allowed to repeat them. The teacher can revise the basic information of the exam, that is, the starting time of the exam and the ending time of the exam. At the same time, the teacher can modify the list of questions in the exam, that is, add questions to the exam and delete the questions in the exam. The teacher can also edit problems in the question library, that is, adding questions to the question library and deleting problems in the question library. When you add a problem, you need to fill in the problem 
description, at least one question option, and the correct problem option.

\section{System Implementation Renderings}

Part of the system implementation effect diagram is as follows,

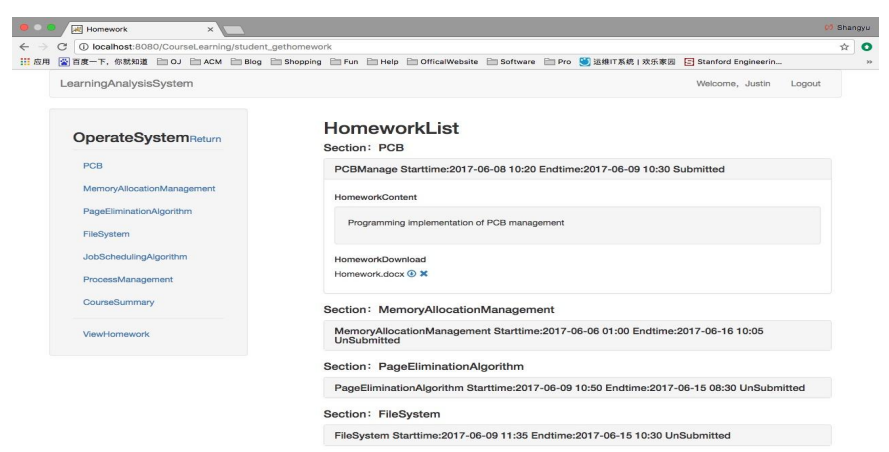

FIGURE III. STUDENT WORK LIST INTERFACE

FIGURE 3 shows students the job list interface, where students can submit assignments.

\section{DATA ACQUISITION AND ANALYSIS OF LEARNING ANALYSIS SYSTEM}

The core part of the learning analysis system is the data acquisition and analysis section. This section will describe the five types of data collection and analysis in detail.

\section{A. Questionnaire Data Acquisition And Analysis}

The questionnaire data is the specific content of the questionnaire, and that is the evaluation of class time, the evaluation of teaching in class, the evaluation of extracurricular teaching and the method of study. Since the above data already exist in the database, the data collection can be directly obtained from the database. The questionnaire data analysis section makes the histogram statistics of the three aspects (the time of class, the content of the teaching in class and the content of extracurricular teaching). As shown in FIGURE 4, the first knowledge point questionnaire in the first section of the class in the operating system leaf teacher.

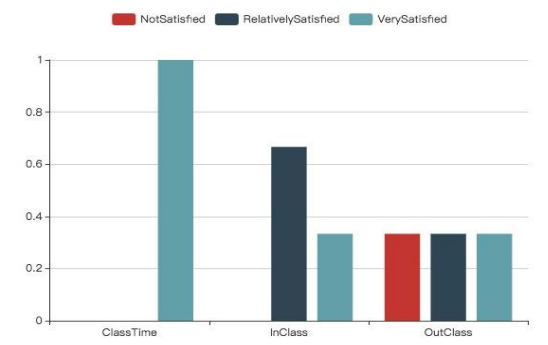

FIGURE IV. QUESTIONNAIRE DATA STATISTICS CHART

The horizontal axis of the histogram indicates questionnaire problem, and the vertical axis indicates the ratio of each option to the total sample. Through the histogram, teachers can quickly see the student's satisfaction of each problem, and they adjust the teaching plan of the course. In Figure 3.1, it can be learned that about $65 \%$ of the students are relatively satisfied with the class time, $55 \%$ of the students are very satisfied with the teaching in class, and $45 \%$ of the students are not satisfied with the extracurricular teaching.

\section{B. BBS Data Acquisition and Analysis}

BBS data includes each student's post number, replies number, obtain the teacher replies number, the post contribution number and the post contribution quantity and so on five targets. Among them, here are some formulas: the number of post contributions $=$ (posting the total number of words + replying the total number of words) / the total number of words in the BBS; the amount of post contribution = (posting amount + replies amount) / BBS total amount of posts.

In the BBS data analysis section, statistical results such as the average, the median, the maximum, the minimum and the standard deviations are calculated for the five indexes mentioned above. At the same time, the post contribution quantity and the post contribution value are used to evaluate the student's active degree, namely active, general active and inactive. The detailed analysis process is as follows:

- Post contribution and post contribution value are for horizontal and vertical coordinates, matching the student to the two-dimensional points in the plane coordinate system.

- Using the KMeans clustering method to classify all the points in the plane coordinate system into three categories

- Obtaining the centers of each cluster, sort the center from the high to the low according to the sum of the horizontal and vertical coordinates, which are set as active, generally active and inactive.

- For each student's class, get the degree of activity of each student.

\section{Video Data Acquisition and Analysis}

Video data includes the action of a student at a certain point in the video, the time for the progress of video length and the date of play. Video data acquisition has certain difficulty, because only when students pause or play when there will be data generation, but it cannot affect the normal video viewing, it is considered to use asynchronous update technology-Ajax. The part of video data analysis for student time action, drawing as shown in FIGURE 5 playing curve, the ordinate for playing schedule, the abscissa for playing track using a twodimensional display, and while drawing the curve, the statistics of the four indicators are displayed: the maximum number of video playing daily, the minimum number of video daily, the median number of video playing daily, the average number of video playing daily. When the curve is bent downward, it indicates that the students are playing back. Teachers can learn the specific learning behavior of students when they watch video through video playing track. Through statistical indicators, it can be seen whether students has difficulty in learning a knowledge point. 


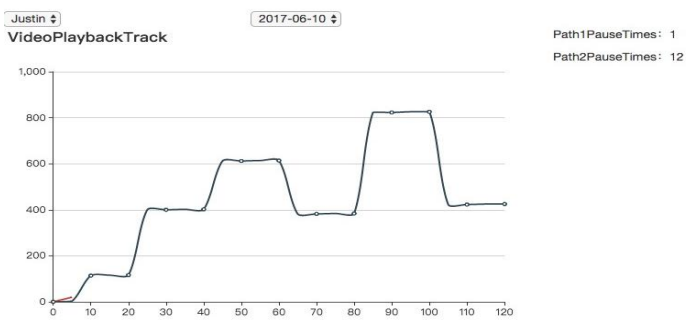

FIGURE V. VIDEO DATA CURVE

\section{Exam Data Acquisition and Analysis}

The exam data included three indicators, such as the time the students submitted for exams, the time spent in the students' exams and the accuracy of the students' exams. The accuracy of the exam is the number of the correct questions / the total number of quizzes. In exam data analysis section, seven statistical results of the maximum, the minimum, the average, the median, the standard deviation, skewness and kurtosis are calculated respectively for the students' time consuming exams and the correct rate of students' exams. Teachers can learn the average student time and exam accuracy from the median, teachers also can anticipate students' exam consuming time, the average exam accuracy and compare them with the actual average time, the actual average correct rate. Teachers can learn the difference between the students in the class by the standard deviation. By the skewness and kurtosis, teachers can be seen in the students' study situation and the degree of concentration, in order to advance adjust their teaching plans to the exam's correct rate of the students with minimal attention to avoid polarization state. In general, this kind of data can reflect the students' knowledge of the situation.

The homework data includes four indicators: the time when students submit their homework, the number of homework submitted by students, the number of homework submitted by students, and the scores of students' homework. In homework data analysis section, there are seven statistical indexes for the number of repeated assignments and the scores of students' work: the maximum, the minimum, the average, the median, the standard deviation, skewness and kurtosis. Teachers can learn from the average whether students have mastered the sections of their homework and pay attention to those who have a low score. From the skewness and kurtosis of students' scores, teachers can learn the differences between students' study situations. In general, this kind of data can reflect the students' mastery of the sections.

\section{CONCLUSION}

The design and analysis platform based on LMS designed in this paper has basically completed its functions, and has realized the design of knowledge system, the division of roles and so on. Through this paper, a analysis platform aimed at students' learning of data can be designed, which puts forward a noveler method and changes the teaching mode of the previous offline.

\section{ACKNOWLEDGMENT}

This work was supported by the National Social Science Fund project (No.17BTQ061), the National Natural Science Foundation of China (No. 61501199), the Projects of the Social Science and Humanity Fund of the Ministry of Education (No. 15YJA880095), the self-determined research funds of CCNU from the colleges' basic research and operation of MOE (No. CCNU17GF0003), and the Hubei Provincial Natural Science Foundation (No. 2017CFB683).

\section{REFERENCES}

[1] Gasevic D, Dawson S, Siemens G, et al, "Let's not forget: Learning analytics are about learning," Techtrends, vol. 59, pp. 64-71, 2015.

[2] M. N. Giannakos, D. G. Sampson, and L. Kidzinski, "Introduction to smart learning analytics: foundations and developments in video-based learning," Smart Learning Environments, vol. 3, pp. 9-12, Dec. 2016.

[3] F. M. Almutairi, N. D. Sidiropoulos, and G. Karypis, "Context-Aware Recommendation-Based Learning Analytics Using Tensor and Coupled Matrix Factorization," IEEE Journal of Selected Topics in Signal Processing, vol. 11, pp. 729-741, Aug 2017.

[4] A. Hernandez-Garcia and M. A. Conde, "Bridging the Gap between LMS and Social Network Learning Analytics in Online Learning," Journal of Information Technology Research, vol. 9, pp. 1-15, Dec. 2016

[5] J. Jovanovic, D. Gasevic, S. Dawson, A. Pardo, and N. Mirriahi, "Learning analytics to unveil learning strategies in a flipped classroom," Internet and Higher Education, vol. 33, pp. 74-85, April 2017.

[6] M. Khalil and M. Ebner, "Clustering patterns of engagement in Massive Open Online Courses (MOOCs): the use of learning analytics to reveal student categories," Journal of Computing in Higher Education, vol. 29, pp. 114-32, April 2017.

[7] Y. B. Hlaoui, F. Hajjej, and L. J. Ben Ayed, "Learning Analytics for the Development of Adapted E-Assessment Workflow System," Computer Applications in Engineering Education, vol. 24, pp. 951-966, Nov 2016.

[8] O. H. T. Lu, J. C. H. Huang, A. Y. Q. Huang, and S. J. H. Yang, "Applying learning analytics for improving students engagement and learning outcomes in an MOOCs enabled collaborative programming course," Interactive Learning Environments, vol. 25, pp. 220-34, 2017.

[9] L. Ali, M. Hatala, D. Gasevic, and J. Jovanovic, "A qualitative evaluation of evolution of a learning analytics tool," Computers \& Education, vol. 58, pp. 470-489, Jan 2012.

[10] [1-3]V. Perumal, R. Butson, P. Blyth, and B. Daniel, "Clinical anatomy e-cases: a five-year follow-up of learning analytics," The New Zealand medical journal, vol. 130, pp. 22-29, Jan 2017.

[11] A. Ramos-Soto, B. Vazquez-Barreiros, A. Bugarin, A. Gewerc, and S. Barro, "Evaluation of a Data-To-Text System for Verbalizing a Learning Analytics Dashboard," International Journal of Intelligent Systems, vol. 32, Feb 2017.

[12] M. Saqr, U. Fors, and M. Tedre, "How learning analytics can early predict under-achieving students in a blended medical education course," Medical Teacher, vol. 39, pp. 757-767, 2017.

[13] R. Ferguson, "Learning analytics: Drivers, developments and challenges," International Journal of Technology Enhanced Learning, vol. 4, pp. 304-17, 2012.

[14] P. Long and G. Siemens, "Penetrating the Analytics in Learning and Education," EDUCAUSE Review, vol. 46, pp. 31-40, Sept.-Oct. 2011.

[15] A. Serrano-Laguna, I. Martinez-Ortiz, J. Haag, D. Regan, A. Johnson, and B. Fernandez-Manjon, "Applying standards to systematize learning analytics in serious games," Computer Standards \& Interfaces, vol. 50, pp. 116-123, Feb 2017. 